\title{
Rehabilitación del segmento anterior de la cavidad oral mediante coronas de disilicato de litio Reporte de un caso
}

López-Llamas AC*, Gaspar-Ovalle KA*, Sepúlveda-Rodríguez A*,

Reynoso-López P**, Díaz-Alfaro L**

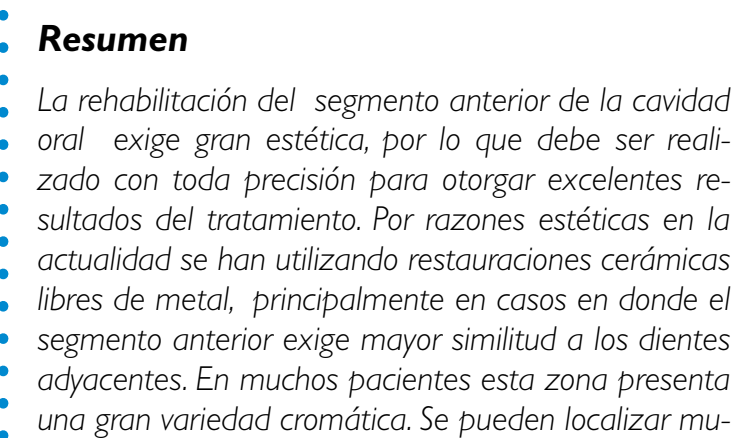

ñones pigmentados o supraestructuras de metal entre otros. Los dientes tratados endodónticamente requieren especial atención para su restauración, debido a la mayor posibilidad de fractura por la eliminación de estructura dental. En este trabajo se presenta un caso clínico de un paciente que requirió rehabilitación del segmento anterior de la cavidad oral mediante coronas de Disilicato de Litio y se discuten sus ventajas y desventajas. LUXMÉDICA, 2011;6(17): 39-43

Palabras clave: endopostes, fibra de vidrio, coronas de disilicato de litio, CAD CAM.

\section{Introducción}

El tipo de restauración en dientes tratados con endodoncia dependerá de los siguientes parámetros: localización del diente en la arcada, morfología radicular, grado de destrucción coronaria, estrés oclusal, y su función como muñón para una prótesis fija o removible. El parámetro más importante es la localización de la pieza den-

Estudiante de la Maestría en Ciencias Biomédicas área Rehabilitación Bucal, del Centro de Ciencias de la Salud, UAA. Dirección Avenida Universidad 940, Ciudad Universitaria Correo electrónico llamas_59@hotmail.com

** Profesores de la Maestría en Ciencias Biomédicas Area de Rehabilitación Bucal, UAA.

Fecha de recibido: 6 de octubre 2010

Fecha de aceptado: 30 noviembre 2010 
tal en la arcada, ya que depende directamente de la carga oclusal que esta tendrá que soportar. ${ }^{1-3}$.

No todos los dientes tratados endodónticamente requieren de postes y coronas, los dientes despulpados anteriores con buena estructura coronal remanente, pueden ser tratados de manera conservadora por medio de una resina. El concepto de que todos los dientes tratados endodónticamente deben ser rehabilitados con poste y corona ya no es vigente como lo demuestran estudios recientes. ${ }^{3-5}$.

Los postes prefabricados han incrementado su popularidad debido a su fácil colocación. Los criterios para obtener resultados favorables son: una longitud adecuada que abarque dos tercios del conducto radicular y forma paralela, ya que aumenta la resistencia y mejor transmisión de fuerzas a través de la estructura radicular. 3,6-8.

Los postes prefabricados no metálicos han sido desarrollados como alternativas y existen sistemas comercialmente disponibles y biocompatibles, resistentes a la corrosión, y con alta flexibilidad, de fácil manipulación y sencillos de retirar en caso de retratamiento. ${ }^{9}$.

Existen varios factores que juegan un papel importante para la selección de los postes estéticos prefabricados tales como propiedades biomecánicas, físicas, forma, estética, costo y sensibilidad a la técnica. ${ }^{10}$.

Después de la colocación del poste, se hace la preparación para colocar una corona cerámica de alta estética lo que implica cerámicas libres de metal. Existen diferentes tipos de porcelana (Feldespática, Alumina, Disilicato de Litio, Spinell, Zirconia, Circonio) para la realización de la restauración final definitiva, y todas ellas buscan un equilibrio entre los factores estéticos, biológicos, mecánicos y funcionales. Para la selección de la cerámica más adecuada es necesario conocer las principales características de estos materiales, y la elección no debe ser delegada al laboratorista. ${ }^{5,8,10}$.

De acuerdo con la literatura y características en este caso clínico se decidió restaurar las piezas tratadas endodónticamente, con la cerámica de Disilicato de Litio (LS2) (IPS e.max R CAD) que tiene excelentes propiedades mecánicas y de dureza. ${ }^{10-13}$. Ofrece una excelente alternativa para la integración estética por la posibilidad de producir restauraciones translúcidas capaces de imitar el esmalte dental natural, además de las múltiples posibilidades de cementación que apoyan a los resultados finales. Esta cerámica posee algunas propiedades como resistencia, exactitud de ajuste marginal, estética, biocompatibilidad. Se puede utilizar en muñones oscuros, decolorados o supraestructuras metálicas. ${ }^{10}$

\section{Presentación del caso}

Paciente masculino de 23 años de edad y que acudió a recibir atención en la clínica de la Maestría en Rehabilitación Bucal, de la Universidad Autónoma de Aguascalientes. Presentaba gran desajuste marginal en restauraciones previas de metal 
porcelana en los Órganos Dentarios (OD) 11 y 21. A la anamnesis clínica reportó traumatismo deportivo con 10 años de antigüedad, produciéndose fractura coronal en los OD 11, 21 con exposición pulpar, recibiendo atención endodóntica. Posterior al tratamiento de endodoncia se colocaron postes metálicos y coronas de metal porcelana. Al momento de la inspección clínica se reporta desajuste marginal de postes y coronas, inflamación gingival, presencia de fístula en el OD 21 y espacio interoclusal reducido a $0.5 \mathrm{~mm}$ aproximadamente (Fig.1-3). En la toma radiográfica periapical se diagnostican lesiones en los ápices de los OD 11 y 21. La dimensión y nivel óseo se ven aceptables. Se decidió retirar los postes y coronas antiguas, para realizar un retratamiento endodóntico así como la colocación de coronas provisionales.

Se decidió clínicamente la colocación de postes. Se realizó aislamiento absoluto, desobturando el conducto radicular con las fresas del sistema Para Post Fiber White (Coltene-Whaledent), limpiando el conducto con solución CONCEPSIS (Chlorhexidine-Ultradent), lavando y secando con puntas de papel y aire. Posteriormente se aplicó ácido ortofosfórico (Total etch- Ivoclar Vivadent) por 15 segundos, se lavó por un minuto y se secó para dejar libre de ácido. Se colocaron tres capas de adhesivo (Excite- Ivoclar-Vivadent) y se fotopolimerizó por 20 segundos al término de la última capa. Posteriormente se utilizó el adhesivo Rely X U100 (3M ESPE), introduciéndolo al conducto por medio de un léntulo, se colocó el poste y fotopolimerizó por 20 segundos. Se procede a la reconstrucción del muñón con resina Tetric Ceram (Ivoclar). Se realizó el tallado de los dientes dejando terminación de Chámfer y se colocaron provisionales (Fig. 4). ${ }^{1,2}$.

Se tomó la impresión de las preparaciones por medio de la técnica de dos hilos, se utilizó 000 interno y 00 externo (Ultrapack, Ultradent). Para la impresión definitiva se utilizó silicón virtual regular y ligero (Polyvinylsiloxan, Ivoclar Vivadent) con la técnica de dos pasos. Se tomó el color de los dientes con el colorímetro vita 3D máster. Se realizó el mapeo enviándolo al laboratorio como guía (Fig. 5). Se corrió el modelo con yeso tipo IV y se mando al laboratorio para la elaboración de las coronas de disilicato de litio. La cementación de la corona se realizó con el cemento Variolink II (Ivoclar-Vivadent) (Figs. 6 y 7). Con las coronas de Disilicato de Litio, ya cementadas en el paciente se puede observar una condición estética y armonía con los dientes adyacentes. Se logra determinar un adecuado ajuste marginal, así como la ausencia de contactos prematuros que puedan afectar la oclusión del paciente.

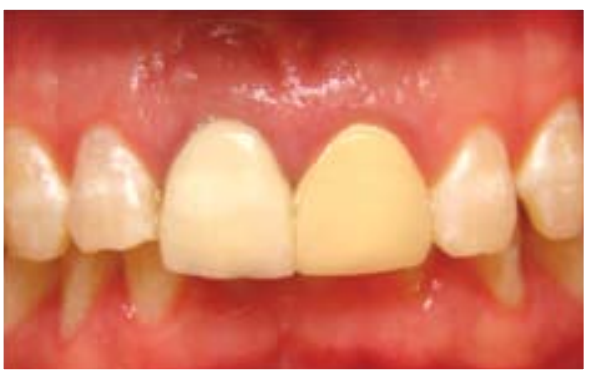

Fig. 1. Restauraciones antiguas de Metalporcelana.

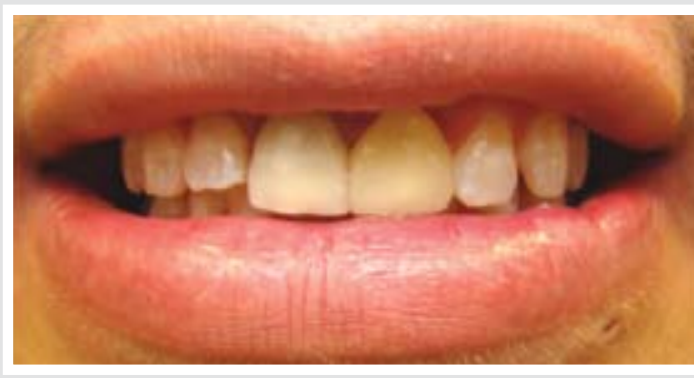

Fig.2. Línea de sonrisa 


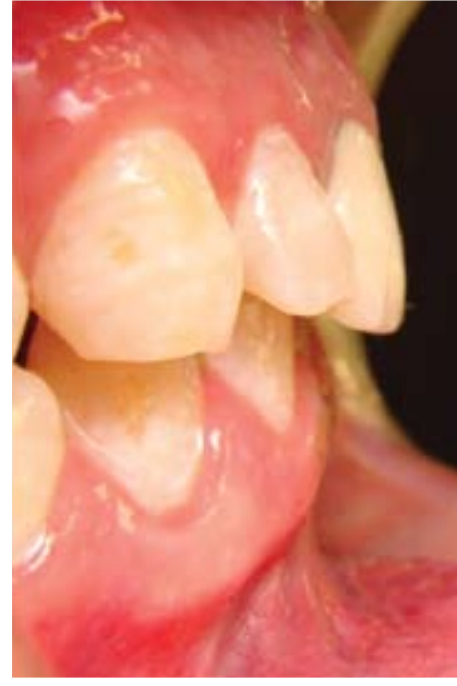

Fig.3. Sobremordida vertical

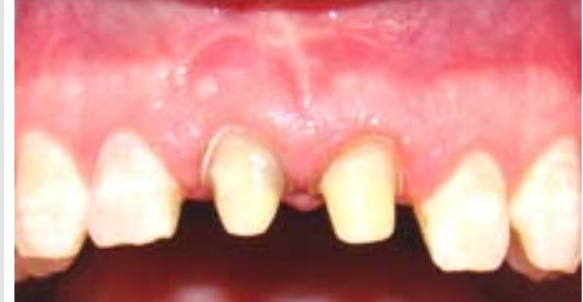

Fig. 4. Muñones con poste de fibra de vidrio.

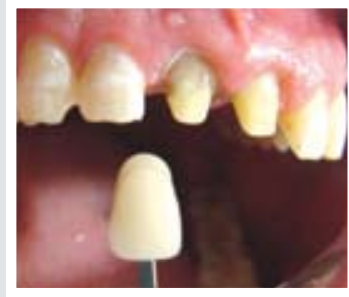

Fig. 5. Toma de color y mapeo individual de cada diente a restaurar.

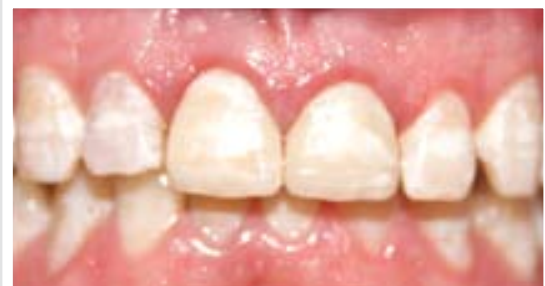

Fig. 6. Restauraciones finales y definitivas a base de Coronas de Disilicato de Litio.

\section{Discusión}

Existen varias opciones para la reconstrucción de dientes tratados endodónticamente. La reconstrucción se puede complicar si existen condiciones clínicas como: ápices abiertos, sobre instrumentación y sobreobturación, fracturas, caries recurrente, resorción interna o externa. 5,9,10

De acuerdo a Duret y colaboradores un poste ideal deberá tener una forma similar al volumen de la dentina perdida una vez preparado el conducto, además de propiedades mecánicas idénticas a la dentina que proveen una adhesión más duradera. EI poste no debe ocasionar tensión mientras es colocado, y lo ideal es que sea mínima la preparación radicular. Varios autores mencionan que los postes translúcidos pueden proveer resultados estéticos. ${ }^{10}$.

En la selección del sistema de postes a emplear, se toma en consideración estética, función, estructura remanente de diente, diseño del diente y propiedades mecánicas. Los postes metálicos han sido utilizados por décadas sin embargo tienen algunas desventajas biológicas y mecánicas como el alto módulo de elasticidad, excesiva reducción dental, pobre retención y fractura radicular. Los postes de fibra de vidrio poseen un módulo de elasticidad similar al de la dentina disminuyendo el riesgo de fractura radicular. 5,13.

Algunas ventajas del uso de postes de fibra de vidrio son: la parte coronaria del diente puede ser reconstruida con resina, los postes contienen partículas de vidrio que conducen la luz que provee una buena polimerización de los cementos de resina, un módulo de elasticidad de $40 \mathrm{GPa}$, considerando que el módulo de elasticidad de la dentina es de $18.6 \mathrm{GPa}{ }^{14-17}$.

Los postes de fibra de vidrio poseen propiedades mecánicas más parecidas a la dentina. Reportes previos en la literatura de postes de fibra de vidrio revelaron la reducción en el riesgo de fracturas radiculares especialmente verticales u oblicuas sobre el tercio medio o apical de las raíces. ${ }^{13,16,17}$

Para solucionar la situación de nuestro paciente elegimos las coronas vitrocerámicas de disilicato de litio ( LS2 ) ( IPS e. max CAD) que ofrecen una mayor resistencia a la fractura y pueden ser fresadas, tienen una dureza aproximada de 440 Mpa y se puede utilizar para fabricar restauraciones unitarias tanto para el segmento anterior como posterior. ${ }^{11}$. 
Este material nos aporta la ventaja de la dureza, muchas veces imprescindible por la falta de espacio interoclusal mínimo de $1.5 \mathrm{~mm}$ que es difícil de lograr en ciertos tratamientos. ${ }^{12}$.

La preparación dental para restauraciones libres de metal se asemeja a la preparación que se diseña para prótesis metal-cerámica no obstante algunos autores matizan especificaciones como reducciones oclusales de 1.5 a $2.0 \mathrm{~mm}$ y axiales de 1 a $2 \mathrm{~mm}$ o un estrechamiento mínimo de $4^{\circ}$ para que pueda ser detectado por el scanner ${ }^{12}$. En ciertas instancias pueden lograrse preparaciones más conservadoras y las restauraciones con disilicato de litio pueden elaborarse tan delgadas como de $0.3 \mathrm{~mm} .{ }^{10}$.

\section{Conclusión}

Los pacientes en la actualidad exigen gran estética en los procedimientos dentales principalmente del segmento anterior, es muy importante la comunicación del clínico con el paciente para hacer la mejor elección del tratamiento y materiales que permitan restauraciones de alta estética, calidad y satisfacción para el paciente.

\section{Bibliografía}

1 Streacker $A B$, Geissberger $M$. The milled Ceramic post and core: A functional and esthetic alternative. The Journal of Prosthetic Dentistry. 2007;98:486-487.

2 Frank P, Peter R. Clinical evaluation of pulpless teeth restored with conventionally cemented zirconia posts: A pilot study. The Journal of Prosthetic Dentistry. 2006;95(4):311-314.

3 Goering A, Mueninghoff L. Management of the endodontically treated tooth. Part I: Concept for restorative designs. The Journal of Prosthetic Dentistry. 1983;49(3):340- 345.

4 Ibraheem-Almansour $M$, Adel-Hammad I. A method to minimize adjustement and enhance passivity of cast post- and- core restorations. The Journal of Prosthetic Dentistry. 2006;95(1):80-81.

5 Maccari PC, Caldas-Cosme D, Mitsuo Oshima H, Henrique-Burnett L, Sadami Shinkai R. Fracture Strength of endodontically treated teeth with flared root canals and restored with different post systems. Journal of Esthetic Restorative Dentistry. 2007;19(1):30-36.

6 Bonfante G, Bazzan-Kaiser O, Pegoraro L. Lins-Do vaIle A. Fracture strength of teeth with flared root canals restored with glass fibre posts. International Dental Journal. 2007; 57(3):153-160.

7 Abdelmageed-Awad $M$, Yousif- Marghalani T. Fabrication of a custom- made ceramic post and core using CAD-CAM technology. The Journal of Prosthetic Dentistry. 2007;98:161-162.

8 Goering AC, Mueninghoff LA. Management of the endodontically treated tooth. Part II: Technique. The Journal of Prosthetic Dentistry. 1983;49(4):491-497.

9 Terry D , Triolo PT, Swift E. Fabrication of Direct
Fiber- Reinforced Posts: A structural Design Concept. Journal of Esthetic and Restorative Dentistry. 2001;13(4):228-240.

10 Maccari $P$, Conceicao $E$, Nunes MF. Fracture Resistance of Endodontically Treated Teeth Restored with Three Different Prefabricated Esthetic Post. Journal of Esthetic and Restorative Dentistry. 2003;15(1):2531.

11 Reco N, Lowe E. Restauraciones libres de metal diseños, Dental Products Report. British Columbia, Información suministrada por Ivoclar Vivadent INC. 2009;20:18-19.

12 Martínez A, Fernández VS, Giner LI, Cortada M. Cerámicas para prótesis parcial fija en el sector posterior. Revisión bibliográfica. DENTUM 2004;4(4):114-117.

13 Miller AW, Tex. Post and core systems: Which one is best? The Journal of Prosthetic Dentistry. 1982;48(1):27-38.

14 Bindl A. Para una mejor transmisión de luz. Coronas de implante CAD/CAM monolíticas de cerámica sin metal basadas en vitrocerámica estética de disilicato de litio. Reflect Dental people for dental people. 2003;09:12-14.

15 Gracia C. Armonizando las expectativas y la estética. Restauraciones estéticas con disilicato de litio ( LS2) en la región anterior. Reflect Dental People for dental people. 2009;3:18-20.

16 Makihira S, Sadamori S. Attaching a magnetic root coping to a fiber-reinforced post. The Journal of Prosthetic Dentistry. 2006;96(5):381-382.

17 Faria e Silva AR, Maia-Casselli D, Bovi-Ambrosano GM, Marcondes-Martins LR. Effect of the adhesive application mode and fiber post translucency on the pushout bond strength to dentin. JOE. 2007;33(9):10781081. 